\title{
UN APORTE A LA EVIDENCIA EMPIRICA DEL EFECTO DE LA EDUCACION SOBRE EL EMPLEO FORMAL
}

María Marta Formichella*

Mara Rojas ${ }^{* *}$

\begin{abstract}
Resumen
El objetivo del presente trabajo es analizar los determinantes de que un trabajador asalariado sea registrado. Principalmente, se investiga el rol que tiene la educación como factor determinante. Para la realización del estudio empírico se utilizan, un modelo PROBIT y datos de la Encuesta de Hogares de Argentina correspondientes al tercer trimestre de 2006. Entre los resultados encontrados se destaca el efecto positivo que tienen la educación, la edad y el género masculino sobre la probabilidad de pertenecer a la categoría estudiada.
\end{abstract}

Clasificación JEL: J0, I2.

Palabras clave: trabajo - informalidad - educación

\begin{abstract}
The objective of the present work is to analyze the determining factors for an employee to be a formal worker. Mainly, the role of education in this regard is studied. For the empirical research a PROBIT model and data from Argentinean Homes Survey corresponding to the Third Trimester of 2006 are used. The most important result is the positive effect that education, age and the male gender have on the probability to belong to the studied category.
\end{abstract}

J.E.L. Classification: J0, I2.

Keywords: labor - informality - education

\section{INTRODUCCION}

La problemática de la informalidad laboral -al igual que otros fenómenos no deseables socialmente, como el desempleo y la exclusión- ha comenzado a ser relevante en las investigaciones acerca de las condiciones de vida de la población. Esto pone de manifiesto la existencia de una preocupación al respecto.

\footnotetext{
* Departamento de Economía, Universidad Nacional del Sur (UNS), Consejo Nacional de Investigaciones Científicas y Tecnológicas (CONICET), e.mail: maryformichella@ yahoo.com.ar

** Departamento de Economía, Universidad Nacional del Sur (UNS), Consejo Nacional de Investigaciones Científicas y Tecnológicas (CONICET) e.mail: mara_rojas05@yahoo.com.ar
} 
Argentina no se encuentra ajena a estos nuevos fenómenos sociales, por ello, aparece como interesante abordar esta temática. En este trabajo, el objetivo es analizar el rol de la educación acumulada por una persona como determinante de su participación en el mercado laboral formal.

Para ello se aplicará un modelo econométrico PROBIT que permitirá analizar la influencia del nivel educativo de un individuo sobre su probabilidad de ser un trabajador asalariado registrado o no registrado. Se utilizarán datos de la Encuesta Permanente de Hogares (EPH) correspondientes al tercer trimestre de $2006^{1}$ y se analizará el caso de los trabajadores asalariados debido a que dicha encuesta no brinda datos suficientes, en relación a la informalidad, sobre los cuentapropistas o sobre los patrones ${ }^{2}$.

En la primera parte se realiza un análisis de los conceptos necesarios para estudiar el tema. En la segunda parte se presenta una descripción estadística del mercado laboral actual en la Argentina, haciéndose hincapié en la situación de los trabajadores asalariados no registrados. En el tercer apartado se presenta el modelo econométrico PROBIT que se utilizará para cumplir con el objetivo del trabajo.

\section{CONCEPTOS BASICOS PARA EL ESTUDIO DEL TEMA}

\section{1. Informalidad}

El concepto de economía informal apareció por primera vez en el año 1970 y lo introdujo el antropólogo Keith Hart, al referirse a los mercados laborales en África (Perlbach et. al. 2002).

Hart, en su informe a la OIT, planteó que en África existía un modelo de trabajo dual, con desigualdades en las oportunidades y basado en la diferenciación del empleo y el autoempleo, donde los autoempleados se denominaban informales y existía una gran variedad de actividades de este tipo (cada una con su propia dinámica). Sin embargo, luego, estas particularidades se perdieron porque el concepto comenzó a usarse en el seno de la Oficina Internacional del Trabajo (OIT) como sinónimo de pobreza.

La OIT utilizó la expresión "sector no estructurado" (actualmente, sector informal) para describir las actividades de los trabajadores pobres que no eran reconocidas, registradas, protegidas o reguladas por la autoridad pública $\left(90^{\circ}\right.$ Conferencia Internacional del Trabajo, OIT, 2002). Esto ocurrió en el año 1972, en el informe que dicha institución realizó sobre Kenia y cuyo importante aporte conceptual consistió en destacar que el problema de empleo en los países menos desarrollados no se centraba tanto en el desempleo, sino en los ocupados que poseían un ingreso muy bajo y eran denominados trabajadores pobres (Tokman, 2001).

\footnotetext{
${ }^{1}$ Cabe aclarar que publicaciones más actualizadas de la EPH, por cuestiones institucionales, no resultan lo suficientemente confiables como para basar el trabajo en ellas.

${ }^{2}$ Se optó por trabajar con la categoría ocupacional "asalariados" y no con los "cuentapropistas" o "patrones", debido a que no hay datos sobre el registro de estas dos últimas categorías. Además, si bien podría realizarse una estimación al respecto, siguiendo los lineamientos de la Organización Internacional del Trabajo (OIT) (ver apartado número uno), dicha estimación podría no ser precisa. Al mismo tiempo, tampoco se tomaron en cuenta los "trabajadores familiares sin remuneración" (trabajadores informales por definición-ver apartado uno).
} 
De esta manera, la informalidad se incorporó a las ciencias sociales cuando éstas tuvieron que explicar la existencia y el aumento de grandes sectores de la sociedad, que no tenían éxito a la hora de integrarse en los nuevos espacios ofrecidos por el mercado de trabajo urbano, que eran, y lo son, el mecanismo principal de integración social y económica de la población (Carpio et. al, 1999).

Portes (1999), siguiendo a Feige (1990) y a Castells y Portes (1989), propone las siguientes definiciones: "el sector informal está formado por aquellas acciones de los agentes económicos que no adhieren a las reglas institucionales establecidas o que no entran bajo su protección", o "el sector informal esta formado por todas las actividades redituables que no están reguladas por el Estado en entornos sociales en los que sí están reguladas actividades similares".

Entonces, dentro del mercado laboral informal conviven dos situaciones claramente diferentes:

a. Por una parte, las economías de "subsistencia", que surgen como consecuencia de la imposibilidad que tienen ciertos individuos para acceder a un empleo, sobre todo en las grandes ciudades. Ante este problema, aparece la búsqueda de sus propias soluciones produciendo o vendiendo un bien o servicio que les de la posibilidad de tener un ingreso que le permita sobrevivir (Tokman, 2001). La mayor parte de estos emprendimientos quedan al margen de la legalidad ${ }^{3}$ y del sistema impositivo (economía "en negro"). Sin embargo, no se puede asegurar que toda actividad que surja con el fin de la supervivencia sea marginal o se encuentre desconectada del sistema.

b. Por otro lado, existen los trabajadores no registrados que desarrollan sus actividades en entidades públicas o privadas pertenecientes al sector formal, es decir que no es exclusivo de la economía informal quedar fuera del marco regulatorio vigente ${ }^{4}$. Perlbach et. al (2002) expresan que las empresas que toman este camino buscan disminuir sus costos laborales.

Sin embargo, lo que más se observa en la realidad es la existencia del cumplimiento de ciertos requisitos en el registro pero no todos. Esto sucede principalmente en países con poca disciplina fiscal y escasa capacidad de control (Tokman, 2001). Un ejemplo es el caso de los trabajadores registrados que perciben una parte de su salario de manera formal y el resto del mismo no, viéndose en consecuencia, disminuidos los aportes jubilatorios y de cobertura de salud que corresponden al trabajador y al empleador, respectivamente.

\section{I.2. Precariedad}

Si bien existe cierta relación entre informalidad y precariedad, ya que generalmente gran proporción de los trabajadores informales poseen problemas de desprotección laboral ${ }^{5}$, son dos conceptos diferentes.

El empleo precario se define por la ausencia de dos elementos fundamentales que están presentes en los empleos típicos (también llamados normales, regulares o protegidos): la

\footnotetext{
${ }^{3}$ Cabe aclarar que al decir que quedan al margen de la legalidad se esta haciendo referencia a que no entran en el marco regulatorio e impositivo vigente, no a que el producto que se comercializa sea ilícito (como el caso de drogas, prostitución o juego ilegal).

${ }^{4}$ Esta realidad se enmarca en lo que Tokman (1992) y Tokman y Klein (1996) (Tokman, 2001) llaman "áreas grises".

${ }^{5}$ Ver los resultados empíricos más delante.
} 
estabilidad en el empleo y la cobertura social (aportes jubilatorios y obra social) (Beccaria, Carpio y Orsatti, 1999). También puede considerarse la ausencia de otros beneficios como los siguientes: seguridad en el trabajo, seguro por accidentes de trabajo, cobertura sindical, vacaciones pagas, aguinaldo, días por enfermedad pagos, condiciones de higiene laboral, entre otros.

La precariedad laboral refleja la menor calidad de los puestos de asalariados, tanto en la economía formal como informal (Beccaria et. al., 1999). Por ejemplo, las modalidades de contratación nuevas en la Argentina, fruto de las reformas laborales, si bien se encuadran en un marco legal poseen características de mayor precarización del trabajo. Esto porque su duración es determinada, porque el empleo es inestable o porque existe una disminución (legal o de hecho) del grado de protección laboral y social (Tokman, 2001).

Este tipo de contrataciones no están al margen de la regulación vigente. Sin embargo los individuos ven disminuido su bienestar por alguna o más de las siguientes vías: no poseen seguro por accidentes de trabajo, para acceder a cobertura de salud deben afrontar el cien por ciento de los gastos, no reciben aportes jubilatorios por parte de sus empleadores, su trabajo posee fecha de finalización, entre otras cuestiones. Ejemplos de este tipo son las becas, los contratos de pasantías y los planes de empleo oficiales, entre otros.

Por último, cabe destacar los problemas que ocasiona la existencia de individuos no registrados. En principio, este fenómeno implica que en un futuro, cuando dichos individuos ya no puedan formar parte del sistema productivo, no tendrán los ingresos necesarios para poder subsistir, ya que no han realizado los aportes jubilatorios correspondientes. De esta manera, en el futuro estarán excluidos del consumo, estarán afuera de la cobertura de salud y pasarán a incrementar la demanda de bienes y servicios al Estado. Esto resulta particularmente preocupante en países como la Argentina, en el que el $42 \%$ de los trabajadores asalariados no están registrados.

Otro problema grave de exclusión que sufren los trabajadores informales tiene que ver con el acceso a la salud, ya que prácticamente la totalidad de los mismos no posee cobertura $(96,2 \%)$. Nuevamente, esto se manifiesta en una mayor demanda en los centros asistenciales públicos.

Roca y Moreno (1999) plantean que la desprotección social no sólo trae aparejado el problema de que los trabajadores no gozan de los beneficios sociales, sino que produce graves problemas en el financiamiento del sistema de seguridad social. Además, el hecho de que se reduzcan los ingresos ocasiona una disminución en la calidad de las prestaciones, al mismo tiempo los sindicatos pierden poder de negociación por la disminución en el número de afiliados y aumenta la desprotección de los asalariados. Así, se va conformando un amplio conjunto de población que queda al margen de los servicios básicos. Estos autores afirman que en el marco de un alto desempleo abierto, la creación de empleo asalariado en condiciones precarias es justificable para combatir la pobreza. Sin embargo, también rescatan que desde la óptica de la seguridad social fundada en el cofinanciamiento empleador-empleado, donde el monto esta basado en el salario del trabajador (sistema bismarckiano), el empleo precario representa mayores niveles de exclusión presente y futura.

El problema de la desprotección social se ve agravado por los bajos ingresos. En el gráfico $\mathrm{N}^{\mathrm{o}} 1$ queda de manifiesto que los deciles más bajos de ingresos concentran la mayor 
proporción de asalariados no registrados, mientras que a medida que se avanza hacia deciles más altos aumenta la proporción de trabajadores registrados.

El mayor número de trabajadores registrados se observa en los deciles 8-10 (43.2\%) de la muestra, mientras que sólo el 5\% de los no registrados pertenece a esos estratos de ingresos. Al mismo tiempo, el mayor porcentaje de trabajadores no registrados pertenece a los deciles 13 , siendo de tan solo un $4 \%$ la participación de los asalariados registrados en dichos deciles. Por lo tanto, se infiere que los trabajadores formales poseen un nivel de ingresos superior a los trabajadores informales.

Gráfico $\mathrm{N}^{\mathrm{o}}$ 1: Asalariados no registrados por grupo de deciles de ingresos

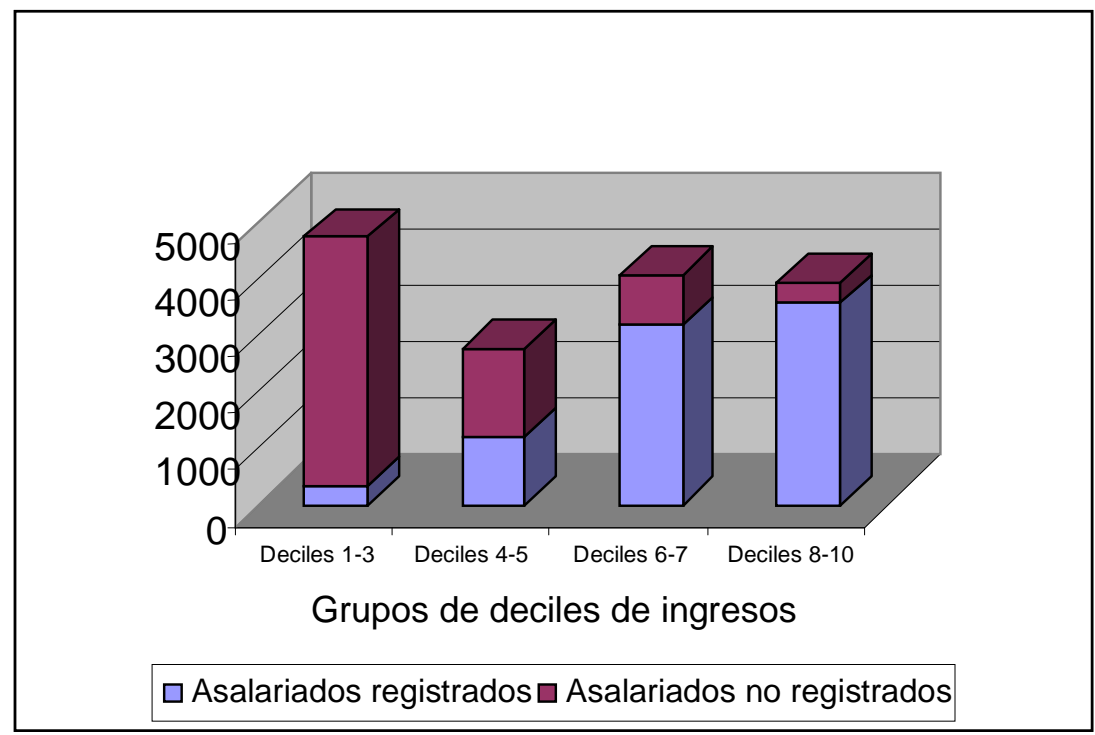

Fuente: Elaboración propia en base a INDEC. EPH. Tercer trimestre de 2006.

Por último, cabe destacar las dificultades que los trabajadores no registrados poseen en relación al acceso al crédito, especialmente al crédito hipotecario, ya que al participar de la producción de manera informal, no tienen manera alguna de acreditar sus ingresos $\mathrm{y}$, menos aún, sus ingresos futuros y su estabilidad laboral (requisitos indispensables para calificar como receptor de un crédito por un banco). De esta manera, dado que su bajo nivel de ingresos no les permite forjar un ahorro para acceder a la vivienda propia $^{6}$, se ven obligados a alquilar, viéndose disminuido su ingreso disponible y por lo tanto sus posibilidades de consumo.

I.3 Tasa de informalidad y tasa de precariedad

La tasa de informalidad se mide como la proporción de ocupados informales sobre el total de ocupados. Sin embargo, dada la diversidad de formas en que puede presentarse el

\footnotetext{
${ }^{6}$ Ver más adelante (apartado 3) el análisis respecto al ingreso de los trabajadores informales.
} 
empleo informal, y dado que se relaciona con el permanecer al margen de la legalidad, con el "no registro"; se hace difícil poder estimar el número de trabajadores informales. Por ello, la discusión sobre cómo medirlo ha sido amplia a través del tiempo y diversos informes se han escrito sobre el tema ${ }^{7}$.

En términos generales y gracias a la tarea conjunta de diversas instituciones, organismos y bloques regionales (MERCOSUR, CEPAL, Unión Europea y Comunidad Andina, entre otros) se ha avanzado en la armonización de las estadísticas entre los países. Sin embargo, respecto al empleo informal los avances son menores debido a la complejidad del tema ${ }^{8}$, y por ello el trabajo en relación a la mejora de las formas de medición es continuo ${ }^{9}$.

En enero de 1993 se realizó la XV Conferencia Internacional de Estadísticos del Trabajo $(C I E T)$. En su resolución queda caracterizado el sector informal y se explicitan recomendaciones que han sido de gran utilidad para la medición del mismo. Sin embargo, ante la necesidad de avanzar más en el tema, dada la importancia que reviste el sector informal en muchos países, la Comisión de Estadísticas de las Naciones Unidas creo el Grupo Delhi, el cual está formado por un Grupo de Expertos Internacionales en Estadísticas del Sector Informal. El objetivo de este grupo es recopilar y certificar las prácticas nacionales de obtención de datos en el sector informal y desarrollar metodologías para que las mismas se enmarquen en el Sistema de Cuentas Nacionales (SCN93) y en las recomendaciones de la XV CIET.

El grupo Delhi, en su sexta reunión (año 2002) enuncio que el empleo informal incluye:

a. Trabajadores independientes y empleadores que tienen sus propias empresas del sector informal.

b. Trabajadores familiares auxiliares que contribuyen con la propia familia, independientemente de si trabajan en empresas del sector formal o informal.

c. Asalariados que tienen empleos informales, ya sea que son asalariados por empresas del sector formal, empresas del sector informal o son trabajadores domésticos asalariados empleados por hogares ${ }^{10}$.

d. Miembros de cooperativas informales de productores.

e. Personas que producen de bienes por cuenta propia exclusivamente para el uso final de estos por parte de su hogar, como la agricultura de subsistencia o la construcción de su propia vivienda.

\footnotetext{
${ }^{7}$ Entre ellos pueden mencionarse los siguientes: "VI reunión del grupo de expertos en estadísticas del sector informal" (2002), "Sector Informal, Concepto, Medición y Políticas" (2003), "Taller regional "Medición de indicadores del mercado de trabajo' (2006), entre otros.

${ }^{8}$ Esto se refleja al analizar los pedidos de los participantes del Taller Regional "Medición de indicadores del mercado de trabajo" (2006) al cual asistieron funcionarios de los institutos nacionales de estadística, funcionarios de los Ministerios de Trabajo de 18 países de América Latina y representantes de la CEPAL, de la OIT y de otras instituciones. Los países manifestaron que era necesario hacer más explícito el concepto de empleo informal por parte de la OIT, también que se haga una mejor distinción entre: empleo informal, sector informal, economía informal y precariedad laboral. Además, se discutió si las encuestas de hogares eran apropiadas y útiles para medir el empleo informal (esto último fue replicado por el Sr. Hussmanns quien indicó que las mismas son el instrumento más idóneo para medir el empleo informal).

${ }^{9}$ Un ejemplo de esto es que desde junio de 2005, la Oficina Regional de la OIT para América Latina y el Caribe está colaborando con el Observatorio del Mercado de Trabajo de MERCOSUR en un proyecto de armonización de indicadores laborales de los países miembros.

${ }^{10}$ Incluir esta categoría coincide con Tokman (1999), quien enuncia que si se define como trabajador informal a todo aquel que no esta protegido y se acepta que el contrato de trabajo es una manera de protección, la carencia del mismo implica una situación de informalidad.
} 
En Argentina, el organismo proveedor de las estadísticas oficiales -el Instituto Nacional de Estadísticas y Censos (INDEC)- proporciona, por medio de los resultados de la EPH, los datos necesarios para calcular el porcentaje de trabajadores asalariados ocupados no registrados, es decir aquellos que no poseen aportes jubilatorios (categoría (c) de la clasificación Delhi). Mientras que no provee datos suficientes para poder calcular el número total de los trabajadores informales.

Al mismo tiempo, con respecto a la tasa de precariedad laboral, que puede definirse como la proporción de trabajadores precarios en relación al total de trabajadores, nuevamente la EPH solamente brinda datos suficientes como para poder medirla en la categoría ocupacional "Obrero o empleado" (asalariado). No provee información sobre la seguridad social (aportes jubilatorios y obra social), ni sobre ninguna otra condición laboral para los trabajadores independientes.

\section{I.4. Educación: componente esencial del capital humano}

Según las Naciones Unidas (1968) el concepto de educación incluye toda una gama de medios complementarios por los cuales se trasmiten el conocimiento, los valores y las especializaciones y se modifican los patrones de comportamiento.

En el análisis del concepto de educación puede considerarse, por una parte, la educación formal, que es aquella que se encuentra dentro de los parámetros del sistema educativo regular, dividido por etapas en educación inicial, media y superior. Por otro lado, se encuentra la educación no formal, que es aquella que forma parte de la instrucción de los individuos, pero no se encuentra sistematizada (por ejemplo: cursos de idiomas o informática). Por último, cabe mencionar la educación informal, ésta se relaciona con servicios educativos extraescolares (clubes, iglesias, ONG’s, etc.) y con la educación en el seno familiar.

Para terminar de definir a la educación es necesario indicar que es un importante elemento constitutivo del capital humano. Según la literatura económica se define como el acervo de cualidades que están impregnadas en el hombre y que lo hacen más productivo. Al formar parte de la persona no puede ser vendido, sin embargo, según la Teoría de Capital Humano, sí afecta los ingresos individuales. Y como se mostrará posteriormente, también influye en otras condiciones de trabajo que tendrá el individuo.

En la Argentina, la EPH provee información sobre máximo nivel alcanzado en educación formal por los individuos encuestados, pero no ofrece características sobre su grado de educación informal.

\section{MERCADO LABORAL EN LA ARGENTINA EN EL AÑO 2006}

En este apartado se intentará caracterizar el mercado laboral en la Argentina, especialmente la situación de los asalariados no registrados. Esto se hará en función de los datos ofrecidos por la Encuesta Permanente de Hogares continua correspondientes al tercer trimestre de 2006. 
Cuadro 1- Principales indicadores del mercado laboral

\begin{tabular}{|l|l|}
\hline Tasa de actividad & $46.2 \%$ \\
\hline Tasa de empleo & $41.1 \%$ \\
\hline Tasa de desempleo & $11.1 \%$ \\
\hline Tasa de subempleo horario & $13 \%$ \\
\hline Tasa de subempleo horario demandante & $8.9 \%$ \\
\hline Tasa de subempleo horario no demandante & $4.2 \%$ \\
\hline Porcentaje de asalariados no registrados & $41.8 \%$ \\
\hline
\end{tabular}

Fuente: Elaboración propia en base a INDEC. EPH Continua. Tercer trimestre de 2006.

Cuadro 2- Cantidad de ocupados por categoría ocupacional

\begin{tabular}{|l|c|c|}
\hline \multicolumn{1}{|c|}{ Categoría Ocupacional } & Total de trabajadores & Porcentaje \\
\hline Asalariados (empleado u obrero) & 19874 & $75.9 \%$ \\
\hline Cuentapropista & 4874 & $18.6 \%$ \\
\hline Patrones & 1118 & $4.3 \%$ \\
\hline $\begin{array}{l}\text { Trabajadores familiares sin } \\
\text { remuneración }\end{array}$ & 324 & $1.2 \%$ \\
\hline Total de ocupados & 26190 & $100 \%$ \\
\hline
\end{tabular}

Fuente: Elaboración propia en base a INDEC. EPH Continua. Tercer trimestre de 2006.

Tal como muestra el cuadro $\mathrm{N}^{\mathrm{o}} 2$, el porcentaje mayor de los ocupados pertenece a la categoría "asalariado". En este trabajo se calculó la tasa de informalidad únicamente para dicha categoría debido a que, tal como se ha explicado anteriormente, no se dispone de la información necesaria para las categorías "cuentapropistas" y "patrones" y los trabajadores familiares sin remuneración son informales por definición (entonces su tasa de informalidad es del 100\%).

Cuadro 3 - Ocupados por nivel educativo según categoría ocupacional

\begin{tabular}{|l|l|l|l|l|l|l|l|}
\hline $\begin{array}{l}\text { Categoría } \\
\text { Ocupacional }\end{array}$ & $\begin{array}{l}\text { Sin } \\
\text { instrucción }\end{array}$ & $\begin{array}{l}\text { Primaria } \\
\text { Incomp. }\end{array}$ & $\begin{array}{l}\text { Primaria } \\
\text { Comp. }\end{array}$ & $\begin{array}{l}\text { Secundaria } \\
\text { Incompleta }\end{array}$ & $\begin{array}{l}\text { Secundaria } \\
\text { Completa }\end{array}$ & $\begin{array}{l}\text { Superior y/o } \\
\text { Univ.Incomp. }\end{array}$ & $\begin{array}{l}\text { Superior } \\
\text { y/o Univ. } \\
\text { Completo }\end{array}$ \\
\hline Patrón & $3,1 \%$ & $1,9 \%$ & $2,8 \%$ & $3,1 \%$ & $4,5 \%$ & $4,3 \%$ & $7,8 \%$ \\
\hline $\begin{array}{l}\text { Cuenta- } \\
\text { propista }\end{array}$ & $35,4 \%$ & $26,6 \%$ & $23 \%$ & $18,9 \%$ & $17,2 \%$ & $15 \%$ & $14,1 \%$ \\
\hline Asalariado & $59,6 \%$ & $69,9 \%$ & $73,2 \%$ & $76,2 \%$ & $77,2 \%$ & $78,7 \%$ & $77,7 \%$ \\
\hline $\begin{array}{l}\text { Trabajador } \\
\text { familiar sin } \\
\text { remuneración }\end{array}$ & $1,9 \%$ & $1,5 \%$ & $0,9 \%$ & $1,8 \%$ & $1,2 \%$ & $1,9 \%$ & $0,4 \%$ \\
\hline Total & $100 \%$ & $100 \%$ & $100 \%$ & $100 \%$ & $100 \%$ & $100 \%$ & $100 \%$ \\
\hline
\end{tabular}

Fuente: Elaboración propia en base a INDEC. EPH Continua. Tercer Trimestre de 2006. 
Sin embargo, cabe destacar que al descartar a los trabajadores independientes se está dejando de lado a una alta proporción de individuos de baja calificación. Tal como lo muestra el cuadro $\mathrm{N}^{\mathrm{o}} 3$, la proporción de individuos cuentapropistas baja notablemente a medida que aumenta el estrato de nivel educativo analizado. Esto puede deberse a que es más difícil lograr ser empleado para los individuos de baja calificación, por lo tanto deben buscar otra manera de subsistir.

Lo planteado en el párrafo anterior es coincidente con la Teoría de "Hipótesis del procedimiento oculto de selección" de Keneth Arrow (1973) y Michael Spence (1973). Estos autores desarrollan el supuesto de que, ante la falta de información completa y perfecta a la hora de conocer la productividad marginal de los postulantes a un puesto de trabajo, el nivel académico alcanzado por los individuos es una manera efectiva y utilizada por las empresas para detectar a los trabajadores más calificados. Según ellos el nivel de estudios alcanzado es un determinante muy fuerte para las empresas a la hora de estudiar la calificación de un trabajador.

Cabe destacar que las regiones Pampeana y Patagónica son las que menor tasa de no registro poseen, si bien son tasas altas (ver cuadro $\mathrm{N}^{\mathrm{o}} 4$ ). Por otro lado, las que en peor situación laboral se encuentran son las regiones Noroeste y Noreste.

Cuadro 4 - Asalariados sin registro por región

\begin{tabular}{|l|l|l|l|l|l|l|}
\hline Tasas & Noroeste & Noreste & Cuyo & GBA & Pampeana & Patagónica \\
\hline Tasa de no registro & $49,9 \%$ & $49,6 \%$ & $47,3 \%$ & $42,3 \%$ & $39,2 \%$ & $26,6 \%$ \\
\hline Tasa de desempleo & $10.1 \%$ & $5.8 \%$ & $4.7 \%$ & $11.3 \%$ & $10.3 \%$ & $8 \%$ \\
\hline Tasa de subempleo & $10.2 \%$ & $9.4 \%$ & $10.9 \%$ & $12.1 \%$ & $9.9 \%$ & $5.7 \%$ \\
\hline Tasa de subempleo demandante & $7.7 \%$ & $5.9 \%$ & $8 \%$ & $7.7 \%$ & $7.4 \%$ & $3.6 \%$ \\
\hline $\begin{array}{l}\text { Porcentaje de individuos } \\
\text { económicamente activos que } \\
\text { demandan empleo. }\end{array}$ & $17.8 \%$ & $11.7 \%$ & $12.7 \%$ & $19 \%$ & $17.7 \%$ & $11.6 \%$ \\
\hline
\end{tabular}

Fuente: Elaboración propia en base a INDEC. EPH Continua. Primer trimestre de 2006.

De este modo, luego de analizar en términos generales el mercado laboral de la Argentina, y antes de pasar al desarrollo del modelo econométrico, resulta interesante caracterizar la situación del grupo a estudiar, es decir de los ocupados asalariados no registrados.

Con respecto al sexo, en principio se observa que el conjunto de trabajadores no registrados está conformado prácticamente en igual proporción por hombres $(50,4 \%)$ y mujeres $(49.6 \%)$. Sin embargo, tomando los dos subgrupos por separado (varones y mujeres) se observa que la tasa de no registro es mucho mayor en el grupo femenino (46.6\%) que en el masculino $(37.9 \%)$.

En relación a la edad, se observa que las edades extremas son las que mayor tasa de no registro poseen. Esto puede verse en le siguiente cuadro:

\footnotetext{
${ }^{11}$ La tasa de no registro se define como el cociente entre el total de trabajadores asalariados no registrados y el total de trabajadores asalariados.
} 
Cuadro 5 - Tasa de no registro según edad

\begin{tabular}{|l|l|}
\hline Edad & Tasa de no registro \\
\hline $10-13$ & $100 \%$ \\
\hline $14-24$ & $64.8 \%$ \\
\hline $25-44$ & $37.7 \%$ \\
\hline $45-64$ & $32.8 \%$ \\
\hline 65 y más & $55.7 \%$ \\
\hline
\end{tabular}

Fuente: Elaboración propia en base a INDEC. EPH Continua. Tercer trimestre de 2006.

Con respecto al tipo de empresa (estatal, privada o mixta) y a su tamaño (pequeña, mediana o grande) ${ }^{12}$, se observa que la mayor tasa de no registro está en las empresas mixtas y en las pequeñas. Roca y Moreno (1999) expresan que el no registro es una característica típica de los microestablecimientos.

Cuadro 6 - Asalariados sin registro según tipo de empresa

\begin{tabular}{|l|l|l|l|}
\hline \multirow{2}{*}{ Tasa de no registro } & Estatal & Privada & Mixta \\
\cline { 2 - 4 } & $19.2 \%$ & $50.5 \%$ & $71.6 \%$ \\
\hline
\end{tabular}

Fuente: Elaboración propia en base a INDEC. EPH Continua. Primer trimestre de 2006.

Cuadro 7 - Asalariados sin registro según el tamaño de empresa a la que pertenecen

\begin{tabular}{|l|l|l|l|}
\hline \multirow{2}{*}{ Tasa de no registro } & Pequeña & Mediana & Grande \\
\cline { 2 - 4 } & $82,2 \%$ & $41,7 \%$ & $18,4 \%$ \\
\hline
\end{tabular}

Fuente: Elaboración propia en base a INDEC. EPH Continua. Primer trimestre de 2006.

En relación al nivel educativo, Monza (1998) encontró un sesgo hacia arriba para los ocupados formales y un sesgo opuesto para los ocupados informales. Esto coincide con los datos que en este trabajo se están analizando. A partir de los mismos se observa un doble efecto, por una parte la mayor proporción de trabajadores no registrados no alcanzaron a terminar la secundaria $(61,3 \%)$ por otro lado, si se estudian los datos por grupo según nivel educativo, se observa que la tasa de no registro baja al subir dicho nivel. Esto puede apreciarse con mayor detalle en el Cuadro $\mathrm{N}^{\mathrm{o}} 8$.

\footnotetext{
${ }^{12}$ De acuerdo a Roca y Moreno (1999) las empresas de menos de 5 ocupados se consideran microempresas (empresas chicas), las que tienen entre 5 y 40 ocupados medianas y las que poseen más de 40 empleados grandes. Si bien esta no es la mejor forma de clasificar a las empresas por tamaño, ya que es mas claro hacerlo según su facturación, aquí se toma ese criterio por la disponibilidad de datos a favor del mismo.
} 
Si se realiza el mismo análisis, pero considerando hombres y mujeres por separado se observa que las tasas de no registro son mayores para las mujeres en todos los niveles educativos considerados, excepto para el nivel más alto (superior universitario completo), en el que las tasas son prácticamente iguales.

Cuadro 8 - Tasa de no registro según nivel educativo

\begin{tabular}{|l|c|}
\hline \multicolumn{1}{|c|}{ Nivel educativo } & Tasa de no registro \\
\hline Sin instrucción & $74 \%$ \\
\hline Primario Incompleto & $69.4 \%$ \\
\hline Primario Completo & $56.7 \%$ \\
\hline Secundario Incompleto & $54.4 \%$ \\
\hline Secundario Completo & $33.3 \%$ \\
\hline Superior y/o Universitario Incompleto & $37.7 \%$ \\
\hline Superior y/o Universitario Completo & $15.8 \%$ \\
\hline
\end{tabular}

Fuente: Elaboración propia en base a INDEC. EPH Continua. Primer trimestre de 2006.

Cuadro 9 - Tasa de no registro según nivel educativo y género

\begin{tabular}{|l|c|c|}
\hline \multicolumn{1}{|c|}{ Nivel educativo } & $\begin{array}{l}\text { Tasa de no registro } \\
\text { masculina }\end{array}$ & $\begin{array}{l}\text { Tasa de no registro } \\
\text { femenina }\end{array}$ \\
\hline Sin instrucción & $67.9 \%$ & $82.5 \%$ \\
\hline Primario Incompleto & $61.8 \%$ & 82.25 \\
\hline Primario Completo & $45.6 \%$ & $74.8 \%$ \\
\hline Secundario Incompleto & $47.4 \%$ & $67.2 \%$ \\
\hline Secundario Completo & $27.9 \%$ & $40.7 \%$ \\
\hline $\begin{array}{l}\text { Superior y/o Universitario } \\
\text { Incompleto }\end{array}$ & $33.8 \%$ & $41.7 \%$ \\
\hline $\begin{array}{l}\text { Superior y/o Universitario } \\
\text { Completo }\end{array}$ & $15.9 \%$ & $15.7 \%$ \\
\hline
\end{tabular}

Fuente: Elaboración propia en base a INDEC. EPH Continua. Primer trimestre de 2006.

Por último, si se analiza la situación tomando en cuenta si los individuos son o no jefes de hogar, se observa que la tasa de no registro es mayor para quienes no son jefes (49.2\%) que para quienes sí lo son (33.5\%). Esto podría deberse a que los jefes de hogar son más selectivos a la hora de tomar un trabajo. Puede ser que esperen a tener una mejor oportunidad laboral, con mejores condiciones de seguridad social. 


\section{EL MODELO ECONOMETRICO}

Para estimar cuál es la probabilidad de que un trabajador asalariado ocupado esté registrado se utilizó un modelo PROBIT ${ }^{13}$, ya que la variable dependiente es dicotómica y en este caso la regresión probabilística provee un análisis superior a la regresión lineal tradicional ${ }^{14}$. En este tipo de modelo, los coeficientes son estimados por el método de máxima verosimilitud.

Como se mencionó anteriormente, se utilizarán datos de la Encuesta Permanente de Hogares (EPH) correspondientes al tercer trimestre de 2006 para el total de los aglomerados relevados. Se utilizaron variables explicativas dummies referentes a regiones geográficas, tamaño de empresa, sector, variables en relación al nivel educativo, la condición de jefe de hogar y sexo. Además, se utilizó la variable edad medida en años.

Respecto de las regiones geográficas, las variables dummies se construyeron de la siguiente manera: la variable toma el valor 1 si se trata de la región geográfica denominada en la misma y cero en cualquiera de los otros casos. Al contarse con datos para seis regiones diferentes, se construyeron cinco variables dummies. Como se verá más adelante, se encontraron diferencias bastante significativas en la condición de empleo de la población según zona de residencia. Por ello se consideró importante tomarlas como variables explicativas.

En relación al tamaño de la empresa, se construyeron dos dummies. En el primer caso, la variable toma valor uno si se trata de una microempresa (menos de cinco trabajadores), y cero en los demás casos. La segunda variable definida toma valor uno si se trata de una empresa grande (más de 40 trabajadores), y cero en caso contrario. Además, se construyó una dummie tomando de referencia el sector estatal (toma valor uno si la empresa pertenece al Estado y cero si pertenece al sector privado o es mixta).

La variable jefe de hogar toma valor uno si el individuo cumple con el atributo y cero si no lo cumple. Por su parte, la variable sexo se definió de forma similar, tomando valor uno si el individuo es hombre y cero si se trata de una mujer.

El nivel educativo se mide con los años de educación formal. Como la EPH registra el máximo nivel de educación alcanzado, se convirtió esta variable ordinal en una numérica, siguiendo la escala que se utiliza en la literatura referente al tema ${ }^{15}$. La escala es la siguiente: $\sin$ instrucción $=0$; primario incompleto $=3$; primario completo $=7$; secundario incompleto $=9$; secundario completo $=12$; terciario y/o universitario incompleto $=13$; terciario y/o universitario completo $=17$.

\footnotetext{
${ }^{13}$ El software utilizado es el STATA 8.0.

${ }^{14}$ Por ejemplo, con Mínimos Cuadrados Ordinarios (MCO) la predicción no se encontrará necesariamente entre 0 y 1 (valores utilizados al trabajar con probabilidades), y además pueden existir problemas de heteroscedasticidad.

${ }^{15}$ Psacharopoulos, G.; Valenzuela, J. y Arens, M. (1993).
} 
Resultado de la regresión:

probit trab_registrado reg_pat reg_pam reg_cuyo reg_noe reg_gba reg_noa gran_emp micro_emp trab_estatal nivel_educ jef sex edad

Probit estimates

Number of obs $=19874$

LR chi2(12) $=5491.61$

Log likelihood $=-10760.22$

Prob $>$ chi2 $=0.0000$

Pseudo R2 $=0.2033$

\begin{tabular}{|c|c|c|c|c|c|c|}
\hline trab_registrado | & Coef. & Std. Err. & $\mathrm{z}$ & $\mathrm{P}>|\mathrm{z}|$ & [95\% Conf & Interval] \\
\hline reg_pat | & .6615344 & .0421644 & 15.69 & 0.000 & .5788938 & .7441751 \\
\hline reg_pam | & .2546597 & .0365233 & 6.97 & 0.000 & .1830753 & .3262441 \\
\hline reg_noe & -.1039205 & .0441074 & -2.36 & 0.018 & -.1903694 & -.0174717 \\
\hline reg_gba & .2030144 & .0400601 & 5.07 & 0.000 & .124498 & .2815309 \\
\hline reg_noa & -.1002008 & .0388876 & -2.58 & 0.010 & -.176419 & -.0239825 \\
\hline gran_emp & .5637075 & .0540289 & 10.43 & 0.000 & .4578129 & .6696022 \\
\hline micro_emp & -.910545 & .1256937 & -7.24 & 0.000 & -1.1569 & -.6641898 \\
\hline trab_estatal | & .697255 & .0246092 & 28.33 & 0.000 & .6490217 & .7454882 \\
\hline nivel_educ & .1252379 & .0028112 & 44.55 & 0.000 & .119728 & .1307477 \\
\hline jef $\mid$ & .220871 & .0230001 & 9.60 & 0.000 & .1757917 & .2659503 \\
\hline sex & .4562754 & .0220105 & 20.73 & 0.000 & .4131356 & .4994152 \\
\hline edad & .0185656 & .000878 & 21.15 & 0.000 & .0168448 & .0202865 \\
\hline _cons | & -2.537809 & .0595631 & -42.61 & 0.000 & -2.654551 & -2.421068 \\
\hline
\end{tabular}

- prvalue

probit: Predictions for trab_registrado

Confidence intervals by delta method

\begin{tabular}{|c|c|}
\hline & 95\% Conf. Inter \\
\hline & 0.6089 [ 0.6013, \\
\hline $\operatorname{Pr}(y=0 \mid x):$ & 0.3911 [ 0.3836 , \\
\hline
\end{tabular}

Los coeficientes de la regresión muestran, en principio, los signos esperados. Un ocupado asalariado que habita en las regiones Patagónica, Pampeana y Gran Buenos Aires, tiene una mayor probabilidad de encontrarse registrado con respecto a los que habitan en la región Cuyo. Trabajar en las regiones de Noroeste y Noreste, disminuye la probabilidad de localizarse en el mercado formal de empleo en referencia a la región tomada como base.

Trabajar en una empresa grande incrementa la probabilidad de encontrarse registrado, mientras que estar empleado en una microempresa, disminuye esta probabilidad. Esto no es difícil de imaginar en la realidad si tenemos en cuenta cuáles son, muchas veces, las condiciones de precariedad de los pequeños emprendimientos en nuestro país.

Algo que llama la atención es el signo del coeficiente que acompaña a la variable "trabajador estatal". Mucho se ha hablado últimamente de la continua precarización del trabajo estatal en Argentina, incluso los datos provistos por la EPH para trimestres anteriores lo 
corroboran ${ }^{16}$. Sin embargo, la regresión indicaría que existe una mayor probabilidad de registro si el trabajador se encuentra empleado en el sector estatal.

Asimismo, si la persona es jefe de hogar, aumenta su posibilidad de ser asalariado registrado. Esto podría explicarse por la mayor necesidad de poseer un empleo con mejores condiciones (cobertura social, mejor salario, estabilidad laboral, etc.), y por la mayor predisposición de los jefes/as de hogar a esperar y conservar un mejor empleo.

En cuanto a la variable sexo, la condición de mujer incrementa el riesgo de ser un trabajador no registrado. En la literatura empírica ${ }^{17}$ se distinguen cuatro tipos de discriminación laboral: salarial, ocupacional, en las posibilidades de adquisición de capital humano y en las condiciones de empleo. La discriminación salarial explica que, ante iguales condiciones de productividad laboral, la mujer recibe pagos menores que el hombre. Según la teoría de la discriminación ocupacional, las mujeres suelen estar concentradas en actividades para las que están excesivamente calificadas o suele pedírseles una mayor calificación que a los hombres para determinadas actividades. La discriminación por adquisición de capital humano se da cuando las mujeres tienen menor acceso a las posibilidades de incrementar su productividad. Por último, la discriminación por condiciones de empleo existe cuando son las mujeres quienes soportan, en mayor medida, la carga del desempleo o del empleo en condiciones precarias. Sería el caso que puede observarse en este trabajo.

La relación importante que se quiere remarcar es la que existe entre las posibilidades que tiene un individuo de hallarse en un empleo registrado y su nivel educativo alcanzado: sin lugar a dudas, niveles educativos más altos aumentan la posibilidad de encontrar empleos en mejores condiciones. La pregunta es cuán relevante es esta relación.

La regresión muestra que, en general, tomando todas las variables explicativas en sus valores medios, la probabilidad de ser un trabajador no registrado es aproximadamente del $40 \%$. Pero la probabilidad hallada para los hombres se reduce a un $32 \%$ mientras que para las mujeres aumenta a un $49 \%$. Si corremos la regresión para cada uno de los niveles educativos definidos en el modelo, considerando las demás variables en la media, la probabilidad de no estar registrado va desde un $86 \%$ para las personas sin instrucción a un $15 \%$ para las personas con educación superior completa.

Además, sin importar cuál sea el nivel educativo alcanzado, las posibilidades de encontrarse en el mercado formal de trabajo, siempre son menores para las mujeres que para los hombres, aunque esta disparidad se reduce a mayores niveles educativos.

\footnotetext{
${ }^{16}$ Por ejemplo, para el primer trimestre del 2006, la tasa de no registro para las empresas estatales era de $51.3 \%$, mientras que para las empresas privadas era de $23.2 \%$.

${ }^{17}$ Tal tipología es efectuada por Actis Di Pasquale, E. y Atucha, A.J. (2003) en su trabajo "Brechas salariales: discriminación o diferencias de productividad", haciendo una revisión bibliográfica del tema, siguiendo las teorías de Becker (1957, 1976), Phelps (1972), Madden (1975), Bergman (1986), entre otros.
} 
Cuadro 10 - Probabilidad de ser empleado asalariado registrado de acuerdo a la condición de género y al nivel educativo alcanzado

\begin{tabular}{|c|c|c|c|}
\hline $\begin{array}{c}\text { Nivel } \\
\text { educativo }\end{array}$ & $\begin{array}{c}\text { Predicción } \\
\text { general }\end{array}$ & $\begin{array}{c}\text { Predicción } \\
\text { hombre }\end{array}$ & $\begin{array}{c}\text { Predicción } \\
\text { mujer }\end{array}$ \\
\hline 0 & 0.1389 & 0.1865 & 0.0921 \\
\hline 3 & 0.2390 & 0.3032 & 0.1705 \\
\hline 7 & 0.4174 & 0.4943 & 0.3259 \\
\hline 9 & 0.5167 & 0.5934 & 0.4204 \\
\hline 12 & 0.6619 & 0.7297 & 0.5694 \\
\hline 13 & 0.7064 & 0.7695 & 0.6180 \\
\hline 17 & 0.8517 & 0.8922 & 0.7885 \\
\hline
\end{tabular}

Fuente: Elaboración propia en base a INDEC. EPH Continua. Primer Trimestre de 2006.

En cuanto a la condición de región de residencia, manteniéndose todas las demás variables explicativas en la media, las probabilidades de estar registrados son de $80 \%$ para la Región Patagónica, 67\% para la Región Pampeana, 67\% para el Gran Buenos Aires, 61\% para Cuyo y, aproximadamente, 57\% tanto para el Noroeste como para el Noreste, siendo las regiones que presentan peores condiciones de ocupación.

Es interesante observar qué sucede con algunos casos particulares condicionando las estimaciones. Por ejemplo, si un hombre trabaja en una gran empresa y tiene el más alto nivel educativo, su probabilidad de no registro es de un 4\%; sin embargo, con las mismas características pero un nivel educativo mínimo, la probabilidad de no registro aumenta a $63 \%$. Una vez más, la importancia de la educación a la hora de explicar la diferencia entre la posibilidad de hallarse en el mercado formal de trabajo o el informal, queda en evidencia. Si la persona, en este caso, es mujer y trabaja en una empresa grande, con el máximo de educación a alcanzar, su probabilidad de no registro es de 9\%; y si, bajo las mismas condiciones posee un nivel mínimo de educación, la probabilidad de no registro es de 78\%. En ambos casos, las probabilidades de no registro son mayores que para los hombres.

Por otra parte, cabe preguntarse cómo determina la condición de jefe de hogar, la posibilidad de registro laboral. En el cuadro 12 se observa (para ambos sexos y para los niveles educativos considerados) que la probabilidad de estar registrado es mayor para los individuos jefes que para los no jefes. En todos los casos, la probabilidad de registro es nuevamente mayor para los hombres.

Al mismo tiempo, si el jefe de hogar es hombre y su educación máxima, la probabilidad de hallarse registrado es muy alta $(91 \%)$. Pero si es mujer, la probabilidad de registro baja en casi 10 puntos $(81 \%)$. En las mismas condiciones, pero con los más bajos indicadores de educación, las probabilidades respectivas son del 22 y $11 \%$. Esto puede deberse a que la mujer jefa de hogar habitualmente forma parte de hogares uniparentales, lo que la lleva a ser menos selectiva a la hora de tomar un empleo. 
Cuadro 12 - Probabilidad de ser un trabajador registrado dada la condición de género, de jefe de hogar y nivel educativo.

\begin{tabular}{|r|c|c|c|c|}
\hline \multirow{2}{*}{ Nivel educativo } & \multicolumn{2}{|c|}{ Hombres } & \multicolumn{2}{c|}{ Mujeres } \\
\cline { 2 - 5 } & Jefe & No jefe & Jefe & No jefe \\
\hline máximo & 0.9135 & 0.8732 & 0.8176 & 0.7535 \\
\hline promedio & 0.7242 & 0.6460 & 0.5553 & 0.4674 \\
\hline mínimo & 0.2217 & 0.1618 & 0.1107 & 0.0744 \\
\hline
\end{tabular}

Fuente: Elaboración propia en base a INDEC. EPH Continua. Primer Trimestre de 2006.

\section{CONSIDERACIONES FINALES}

Evidentemente la situación del mercado laboral argentino presenta problemas: con una tasa de desempleo del $11.1 \%$ y de subempleo horario demandante del $8.9 \%$, la demanda insatisfecha de trabajo asciende al $20 \%$, mientras que la tasa de asalariados no registrados es del $41.8 \%$.

En cuanto a las categorías ocupacionales, se observa que la mayor proporción de los trabajadores son asalariados. Sin embargo, dicha proporción aumenta a mayor nivel educativo, mientras que el porcentaje de cuentapropistas disminuye. Esto puede deberse a que las personas con bajo niveles de instrucción tienen mayor predisposición a realizar tareas por cuenta propia ante la creciente selectividad para los puestos asalariados. Este fenómeno, que se ha dado últimamente en nuestro país, se denomina "credencialismo" y hace referencia al hecho de que quienes no tienen título (al menos secundario) tienen posibilidades muy acotadas de trabajos asalariados. Los empleadores, al no conocer la productividad de los futuros trabajadores, utilizan el título como señal de la misma.

El grupo estudiado a lo largo de este trabajo esta formado por los trabajadores asalariados no registrados. Ser un trabajador asalariado no registrado en la Argentina significa no tener acceso a cobertura de salud, al sistema de jubilaciones y pensiones, ni al crédito. Además, implica percibir salarios menores. Todo esto pone en evidencia el grado de importancia de esta problemática.

Se han analizado diversos aspectos en relación a esta situación. Respecto a la edad, la tasa de no registro disminuye desde las edades más bajas hasta la categoría de 65 años y más, donde vuelve a darse un incremento probablemente por reinserción laboral de los ancianos que perciben una baja jubilación y que buscan una nueva fuente de ingresos, o los ancianos que nunca han podido realizar aportes jubilatorios y se encuentran sin cobertura social.

En relación al genero, la tasa de no registro es mayor para las mujeres que para los hombres. Podría hablarse aquí de cierta discriminación por condiciones de empleo, siendo las mujeres quienes soportan el mayor peso de la precariedad laboral. Al mismo tiempo, se observa 
que quienes son jefes de hogar poseen una menor tasa de no registro debido a una mayor selectividad.

Las pequeñas empresas son las que menor nivel de registro laboral poseen. Esto último es coincidente con la idea de que las microempresas poseen mayores dificultades para afrontar los costos laborales. Al mismo tiempo, es más probable ser un trabajador asalariado registrado si se trabaja en empresas grandes o estatales.

Además, de acuerdo a los resultados obtenidos, quienes habitan en las regiones Patagónica, Pampeana y Gran Buenos Aires poseen una mayor probabilidad de encontrarse registrado que quienes habitan en las regiones de Cuyo, Noroeste y Noreste.

El foco de atención del trabajo ha estado puesto en la relación educación-condición laboral. En principio, se observa estadísticamente que los individuos menos instruidos poseen una tasa de no registro más alta. A su vez, según el modelo, niveles educativos más altos aumentan la probabilidad de encontrar empleos en mejores condiciones. Es más, al realizarse el ejercicio de condicionar la estimación en el modelo PROBIT desarrollado, se observó que el determinante más fuerte de la probabilidad de ser registrado es el nivel educativo. Sin embrago, cabe señalar, que el efecto de la educación puede estar sobreestimado al no poder distinguir jerarquías ocupacionales dentro del grupo de asalariados. Si bien en la EPH no se dispone de este dato, sería interesante poder incorporar esta variable al análisis.

En síntesis, la situación del trabajo no registrado se presenta como una grave problemática en nuestro país. El análisis sobre los determinantes de la situación laboral de un individuo es complejo, siendo muchas las variables vinculadas a la problemática. Sin embargo, a la luz de la evidencia presentada, podría establecerse que la educación es un condicionante de relevancia para el acceso al mercado laboral formal.

\section{REFERENCIAS BIBLIOGRAFICAS}

Actis Di Pasquale, E. y Atucha,, (2003), "Brechas salariales: discriminación o diferencias de productividad", en Momento Económico, Buenos Aires, mar-abr, (123), pp. 23- 33.

Beccaria, L., Carpio, J. y Oosatti, A., (1999), Argentina: Informalidad laboral en el nuevo modelo económico, Comp. Jorge Carpio, Emilio Klein e Irene Novacovsky. Informalidad y exclusión social. Fondo de Cultura Económica, SIEMPRO y OIT.

Bermúdez, I., (2006), "Bajó el empleo en negro en todo el país, menos en Capital y Córdoba", Diario Clarín. 14 de Junio de 2006.

Bermúdez, I., (2006), "Dónde trabajan, cómo y cuál es el sueldo de los argentinos con empleo". Diario Clarín. 30 de Abril de 2006.

Bermúdez, I.,(2005), "El empleo en negro no cede: afecta al 47,5\% de los asalariados". Diario Clarín. 11 de Junio de 2005.

Carpio, J.; Klein, E. y Novacovsky, I., (1999), Informalidad y exclusión social. Fondo de Cultura Económica, SIEMPRO y OIT.

CEPAL, (2003), El avance en la implementación del sistema de cuentas nacionales 1993 en América Latina y el Caribe, 2002-2003. 
Chitarroni, H., (2002), Equidad del gasto educativo: una mirada desde la oferta y la demanda en el nivel medio, documento del Consejo Nacional de Coordinación de Políticas Sociales, Presidencia de la Nación.

Filmus, D., Miranda, A. y Zelarrayan, J., (2003), La transición entre la escuela secundaria y el empleo: los recorridos de los jóvenes en el Gran buenos Aires. V Congreso de la Asociación Latinoamericana de Sociología del Trabajo (ALAST), La Habana.

Hussmanns, R., (2002) Sexta reunión del grupo de expertos en estadísticas del sector informal (Grupo DELHI). En http://www.oit.or.cr/estad/taller/docs/delhi6pap esp.doc.

INDEC, (2006), EPH Continua. Base Usuaria Ampliada para personas. Tercer trimestre de 2006. En www.indec.gov.ar

INDEC, (2006), Informe de Prensa "Mercado de trabajo: principales indicadores". Marzo de 2006. En www.indec.gov.ar

INDEC, (2006), EPH Continua. Diseño de Registro y Estructura para las bases preliminares Hogar y Personas. Tercer Trimestre. En www.indec.gov.ar

Klein, E,. (1999), Las políticas hacia el sector informal urbano y la OIT: una mirada retrospectiva, Comp. Jorge Carpio, Emilio Klein e Irene Novacovsky. Informalidad y exclusión social. Fondo de Cultura Económica, SIEMPRO y OIT.

Mendicoa, G y Veneranda, L., (1999), Exclusión y marginación social. Secretaría de Desarrollo Social. Espacio Editorial Buenos Aires.

Monza, A., (1998), La evolución de la informalidad en el área metropolitana en los años noventa. Resultados e interrogantes, Comp. Jorge Carpio Emilio Klein e Irene Novacovsky. Informalidad y exclusión social. Fondo de Cultura Económica, SIEMPRO y OIT.

OIT, (1993), Decimoquinta Conferencia Internacional de Estadísticos del trabajo (CIET, En www.ilo.org/public/spanish/standards/relm/gb/docs/gb270/gb-17.htm -20k.

OIT, (2002), El trabajo decente y la economía informal, Conferencia Internacional del Trabajo 90na reunión. Ed. OIT.

OIT, (2005), Políticas para fomento del empleo y trabajo decente. En http://portal.oit.or.cr/index.php/ option=com_staticxt\&staticfile=foro/polit_empleo1.pdf.

OIT, (2006), Taller Regional "Medición de indicadores del mercado de trabajo", En www.lamp.itcilo.org/eurosocial-empleo/index.php?s=2\&area=1 - 7k.

Perlbach, I.; Calderón M. y Papi S. (2002), "Clusters aplicados a la informalidad en Mendoza" Anales de la Asociación Argentina de Economía Política.

Pok C., (2001), "La medición del sector informal en la Argentina, Taller sobre mediación del sector informal en Latinoamérica, OIT. www.wiego.org/publications/POK/20SECTOR/20INFORMAL/20Doc.doc.

Portes, A. (1999), La economía informal y sus paradojas Comp. Jorge Carpio, Emilio Klein e Irene Novacovsky, Informalidad y exclusión social, Fondo de Cultura Económica, SIEMPRO y OIT.

Psacharopoulos, G.; Valenzuela, J. y Arens, M., (1993, “Teachers'salaries in Latin American. A comparative analysis" Working papers Series, Technical Department Latin American and the Caribbean. The World Bank.

Ramos Soto, A. y Gomes Brena, A. R., (2006), ¿Qué es la economía informal?”. Observatorio de la economía latinoamericana $N^{\circ} 60$. www.eumed.net/cursecon/ecolat/mx/2006/rsgb-informal.htm.

Roca, E. y Moreno, J. (1999) "El trabajo no registrado y la exclusión de la seguridad social". Compiladores Jorge Carpio, Emilio Klein e Irene Novacovsky. Informalidad y exclusión social,Fondo de Cultura Económica, SIEMPRO y OIT. 
Tokman, V., (1999), El sector informal posreforma económica, Comp. Jorge Carpio, Emilio Klein e Irene Novacovsky. Informalidad y exclusión socia, Fondo de Cultura Económica, SIEMPRO y OIT.

Tokman, V., (2001), De la informalidad a la modernidad, Ed. OIT. 